\title{
Intégration horticulture - élevage dans les systèmes agricoles urbains de la zone des Niayes (Sénégal)
}

Safiétou Touré Fall, Abdou Salam Fall, Ibrahima Cissé, Aminata Badiane, Cheikh Alassane Fall et Maty Ba Diao

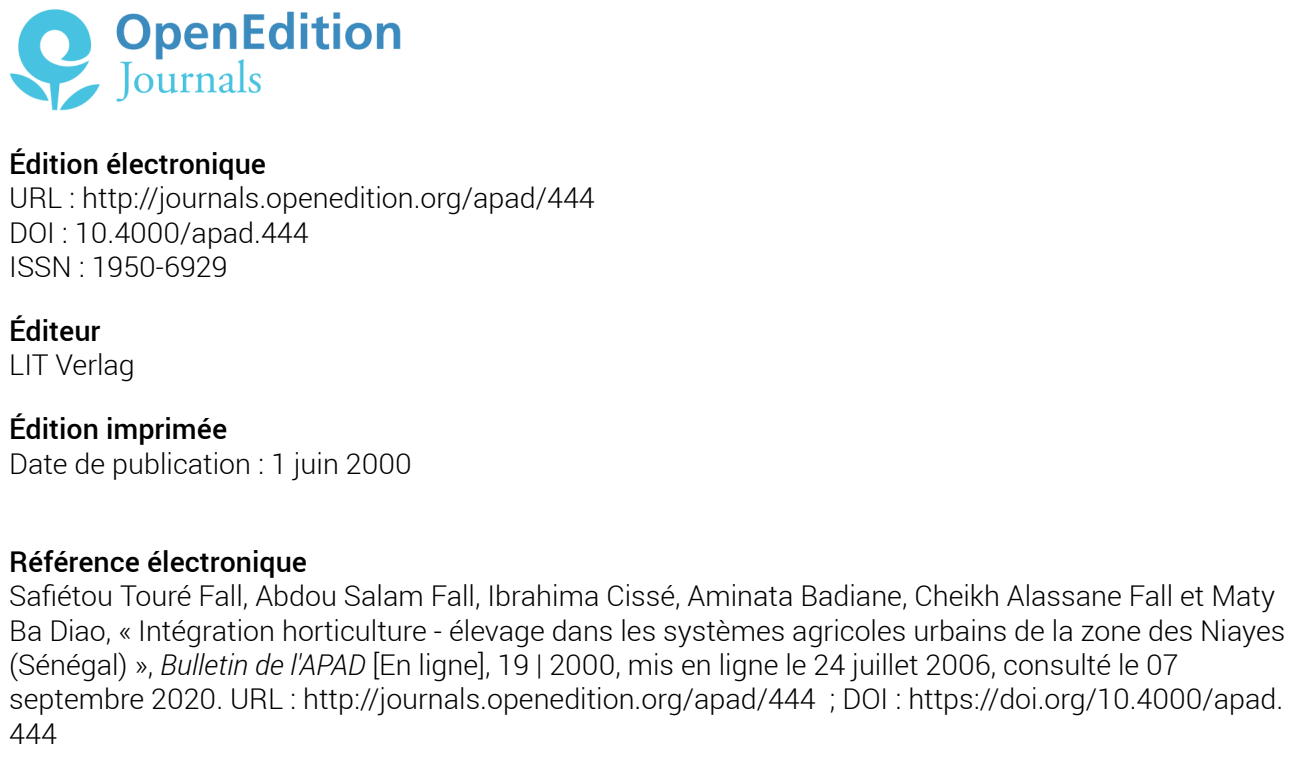

Ce document a été généré automatiquement le 7 septembre 2020

Bulletin de l'APAD 


\title{
Intégration horticulture - élevage dans les systèmes agricoles urbains de la zone des Niayes (Sénégal)
}

\author{
Safiétou Touré Fall, Abdou Salam Fall, Ibrahima Cissé, Aminata Badiane, \\ Cheikh Alassane Fall et Maty Ba Diao
}

1 Les contre-performances de l'agriculture rurale et le déficit alimentaire encouragent depuis plusieurs décennies le développement d'une activité agricole urbaine et péri urbaine dont les principaux domaines sont l'horticulture et l'élevage. La contribution de cette agriculture urbaine à l'approvisionnement des villes est mal connue au Sénégal. Les rares sources disponibles indiquent que Dakar a lui seul prend $40 \%$ de la demande totale de légumes du Sénégal et cette région couvre plus de $60 \%$ de sa consommation en légumes. De même, en fournissant $33 \%$ de la production nationale de poulet, la région de Dakar arrive à satisfaire 65 à $70 \%$ de la demande nationale en poulet (Mbaye et Moustier, 2000).

2 L'agriculture urbaine est très importante dans d'autres villes africaines atteignant près de $70 \%$ en Afrique de l'est alors qu'elle occupe souvent plus de $30 \%$ des populations urbaines. Elle représente donc un élément clé dans la sécurité alimentaire des villes en expansion et constitue un élément régulateur d'un chômage endémique aggravé par l'exode rural.

3 La diversité biologique et la pluralité du système de production des Niayes ont été décrites. Cette diversité biologique n'est pas mise à profit pour améliorer les performances du système de production. Les acteurs n'intègrent pas suffisamment les activités agricoles et pastorales. Peu de producteurs donnent une égale importance à l'élevage comparativement à l'horticulture. Les potentialités qu'offre le recyclage des différents éléments nutritifs de l'horticulture vers l'élevage justifient le développement de technologies dans ce sens.

4 Mais ces activités agricoles ne sont pas sans conséquences sur un environnement urbain marqué par la promiscuité et une compétition entre l'agriculture et l'urbanisation pour l'occupation de l'espace. Au centre de cette problématique, se pose 
avec acuité la question de la gestion de l'espace et des déchets urbains pour l'amélioration du cadre de vie dans les villes. Ainsi, l'intégration des activités agricoles périurbaines apparaît comme une réponse à la pauvreté, un compromis pour concilier les objectifs d'amélioration de l'offre alimentaire et de protection de l'environnement urbain.

Pour améliorer la capacité de l'agriculture urbaine à satisfaire les besoins alimentaires des populations en augmentant sa productivité, les contraintes spatiales imposent alors une intensification de la production. L'utilisation d'intrants chimiques à forte dose semble être une alternative imparable. Mais les risques de détérioration du milieu exigent la prospection d'autres solutions dans le sens d'un recyclage des éléments nutritifs au sein de systèmes mixtes intégrant l'horticulture, l'élevage et les productions halieutiques. Il sera donc important de développer des techniques biologiques d'intégration entre l'horticulture et l'élevage; ce système, faible consommateur d'intrants parce que basé sur le recyclage des déchets, permettra d'améliorer la production en protégeant l'environnement.

6 Cette vision du développement de l'agriculture urbaine est peu répandue au Sénégal. Il existe une minorité d'agriculteurs dits "biologiques" qui s'efforcent de promouvoir des pratiques culturales peu utilisatrices d'intrants chimiques. Ce groupe cherche à se fortifier mais se trouve confronté à un défaut d'encadrement technique et à une insuffisance d'informations et de technologies dans ce domaine. La recherche tente de répondre à cette demande pour contribuer au développement d'un système contraint par l'espace, la démographie et les insuffisances de technologies.

Typologie des systèmes de production dans la zone des Niayes

7 Les Niayes sont avant tout une zone écologique spécifique constituée d'un ensemble de dunes et de dépressions d'âges, de textures et de couleurs différentes. C'est pourquoi, la typologie emprunte à l'écosystème ses différenciations qui se reflètent dans les divers sites de production observables en discontinu dans cette région naturelle. D'autres critères de différenciation comme les spéculations et les types ou techniques d'exploitation sont utilisés.

8 La zone des Niayes présente un système de production horticole assez diversifié lié étroitement à la typologie des sols, à la topographie et surtout à la présence de l'eau dans le milieu. Ainsi on peut déterminer d'est en ouest et du nord au sud le système du dior, des cuvettes, des lacs asséchés, des Niayes proprement dites et des N'diouki.

Le Dior

9 C'est la terminologie locale des sols ferrugineux tropicaux non lessivés qui constituent près de $70 \%$ de toute la région des Niayes allant de Dakar jusqu'à Saint-Louis. C'est le système des dunes ogoliennes. Peu humifères, parfois même très pauvres en matières organiques, ces sols furent longtemps exploités par le système d'agriculture pluviale qui a régressé du fait de la dégradation des conditions pluviométriques. Aujourd'hui, compte tenu de la possibilité d'accès à la nappe qui est à une profondeur variable entre 10 et $30 \mathrm{~m}$ et la possibilité pour certains de se raccorder au réseau de la Société des Eaux (SDE), l'agriculture pluviale a cédé partiellement la place aux cultures maraîchères.

10 Compte tenu de l'ouverture de ce système dunaire, les exploitations maraîchères ont des tailles variables déterminées souvent par la possibilité d'accès à la ressource en eau.

11 Ainsi, les surfaces cultivables peuvent aller de quelques centaines de mètres carrés à des dizaines d'hectares; toutefois les petites exploitations sont largement dominantes 
dans ce système. Les grandes étendues de terres qu'on retrouve dans cette partie des Niayes expliquent la présence de grandes (plus de $20 \mathrm{ha}$ ) et moyennes exploitations ( 1 à 20 ha) notamment dans la région de Dakar (Communauté rurale de Sangalkam), dans la région de Thiès (à Mboro), et dans la région de Saint-Louis (Gandiolais, Dakar-Bango). Dans le dior, les cultures dominantes sont le haricot vert, l'oignon, les choux, la tomate. Ensuite vient la deuxième catégorie de cultures que sont l'aubergine, le jaxatu, le piment, la pastèque, etc. Dans les grandes exploitations, intervient un personnel spécialisé (agronomes, techniciens, spécialistes dans la maintenance des systèmes d'irrigation ou du matériel d'exhaure : motopompe, forage, etc.). Les ouvriers agricoles $\mathrm{y}$ travaillent dont les femmes en particulier pour la récolte. Le système repose essentiellement sur la disponibilité de l'eau de la nappe dont les fluctuations constituent l'un des facteurs les plus contraignants de la production horticole et, une forte utilisation d'engrais minéraux et organiques. Ce système reste très présent dans la région de Dakar, notamment au niveau de la grande Niaye de Pikine entre les dunes séparant deux dépressions où la culture dominante est la salade, et dans la communauté rurale de Sangalcam et, dans les Niayes septentrionales (Lompoul, Potou Gandiolais) où l'oignon reste la principale culture. Des produits de spéculation comme le haricot vert et la tomate sont destinés à l'exportation, tandis que les écarts de tri approvisionnent les marchés nationaux avec Dakar comme principale destination.

Les autres cultures sont acheminées vers les autres marchés. Une partie non négligeable de la production d'oignon, de choux traverse les frontières notamment vers la Mauritanie. L'essentiel de l'élevage intensif, surtout en ce qui concerne la production laitière est implanté dans le dior de même que l'arboriculture fruitière qui y est dominante. Le facteur limitant demeure la profondeur de la nappe phréatique qui peut atteindre de 20 à $30 \mathrm{~m}$. Une autre contrainte réside dans la pauvreté des sols qui nécessitent un apport considérable de fertilisants.

Les Niayes

13 Les Niayes déterminent les dépressions interdunaires caractérisées par des sols très humifères, une faible profondeur, voire même un affleurement de la nappe phréatique et une végétation de type guinéenne. Larges et étalées en longueur au niveau de la presqu'île du Cap-vert, les Niayes se rétrécissent dans la région de Thiès formant de nombreuses mares taries en période sèche et qui s'anastomosent en hivernage. Elles s'égrènent comme des perles au fur et à mesure que l'on remonte vers le nord. Ces dépressions constituent les zones de prédilection des cultures maraîchères.

Compte tenu de leur exiguïté, la taille des parcelles cultivées reste de dimension assez réduite variant de 0,1 à 1 ha. On remarque à ce niveau une forte densification des parcelles et une diversité de spéculations. Les dominantes demeurent les choux, les oignons, les tomates, les aubergines, les jaxatu, les radis, les laitues, les fraises, la menthe, les poivrons, le persil, les poireaux, les concombres. Le site des Niayes est plus concentré dans la région de Dakar (presqu'île du Cap-vert) et dans celle de Thiès (Mboro) où ils forment des espaces caractérisés par une présence quasi permanente de l'eau à la différence des sites dior.

Les Niayes accueillent une partie importante de l'aviculture. Ils viennent comme seconde zone arboricole grâce à la production de papaye, banane, noix de coco, vin de palme, etc. La plupart de la production maraîchère et avicole est orientée vers Dakar, Thiès, Touba, Kaolack, etc. En plus des producteurs locaux dont les familles ont acquis un droit d'usage sur les terres, interviennent massivement des migrants nationaux et 
étrangers en particulier des Guinéens. On y retrouve donc le métayage, le confiage et la location de terre. En plus de l'exiguïté, la salinité des terres par endroits reste un handicap certain sur le système de production. Dans le système de production des Niayes se déterminent également deux types de culture conditionnée par la présence de l'eau de la nappe phréatique affleurante. Ainsi, on a les cultures de décrue au niveau des Niayes de Dakar et de Mboro, et les cultures irriguées localisées dans les parties les plus ex ondées des dépressions.

Dans les Niayes de Dakar notamment la grande Niaye de Pikine et, les Niayes de Mboro les principales cultures de décrue sont les tomates, les patates douces et les oignons, particulièrement à Mboro. L'arboriculture reste importante notamment dans la production de cocos, papayes, bananes corossoles, etc. Les cultures nécessitant une irrigation sont largement dominées par la production de choux sur toute l'étendue des Niayes de Dakar, jusqu'à Saint-Louis. Le système de production dans les dépressions est continu durant toute l'année et, le calendrier de production est plus déterminé par les opportunités du marché que par les conditions météorologiques : ceci est favorisé par l'utilisation de semences améliorées.

Les N'diouki

Ce sont des cuvettes maraîchères localisées sur le système des dunes vives et des dunes semi-fixes (dunes blanches et dunes jaunes). Ils sont présents surtout entre Kayar et le Gandiolais. En effet, la présence de lentilles d'eau douce à faible profondeur a conditionné le développement du maraîchage et, la spécialisation dans la production de carotte et de navet. En outre, il bénéficie d'une bonne protection contre les vents de secteur NNW grâce aux périmètres de reboisement de Casuarina équisétifolia sur tout le long du littoral nord.

Le système de production des N'diouki est beaucoup plus présent dans les Niayes centrales (région de Thiès) et septentrionales (région de Louga et Saint-Louis) et, la taille des exploitations reste de dimension réduite ne dépassant pas 1 ha. Compte tenu de la pauvreté des sols, le système de production des N'diouki nécessite de grands apports en fertilisants minéraux et organiques.

19 La taille des exploitations est plutôt petite en raison de la contrainte majeure qui demeure la remontée du biseau salée (remontée des eaux marines). La production agricole est évacuée vers les principales villes notamment Dakar, Thiès, Saint-Louis, Touba avec le transit vers les marchés relais sur cet axe : Njoxob (keur Abdou Ndoye), Mboro ${ }^{1}$, Jogo, Potu. La Mauritanie reçoit une part non négligeable de cette production notamment celle de choux et de manioc. Les acteurs sont les populations locales parmi lesquelles des pêcheurs pratiquant le maraîchage, des peuls, et d'autres ethnies : wolof, etc.

Aux contraintes relevées plus haut, s'ajoute l'enclavement de ces zones difficiles d'accès (routes impraticables, pistes sablonneuses, déplacements de la production faits en marée basse le long de la plage). L'élevage est quasi inexistant mis à part quelques petits ruminants (ovins, caprins) détenus par des ménages.

Les vallées et lacs asséchés

21 Ce sont d'anciens réseaux hydrographiques devenus secs par la péjoration climatique. Ils sont plus concentrés dans la région de Thiès (Mbawaan : axe Somone - lac Tanma, et au niveau de la région de Dakar (Youi, Mbeubeusse, Malika, etc.). Autrefois parsemée de 
lacs pérennes parallèles à la côte, la région des Niayes par la dégradation des conditions climatiques et pluviométriques. a vu nombre de ses lacs asséchés.

Aujourd'hui, les maigres écoulements de surface observables dans cette zone ne sont que temporaires et tributaires d'une bonne année pluviométrique. Cependant, ces lacs asséchés sont exploités par les maraîchers. Ce système d'exploitation est spécifique au niveau des régions de Dakar et de Thiès où l'on note une concentration de vallées asséchées. La production dominante dans ces systèmes est le chou et la tomate. Secondairement, on y retrouve l'aubergine, le jaxatu, le piment, la tomate, le concombre, la courgette, etc. La production est destinée aux marchés des villes : Dakar, Thiès, etc. Les acteurs sont pour la plupart des migrants provenant du bassin arachidier mais aussi de Guinée. Le facteur limitant est le sel qui envahit les terres et la nappe phréatique. L'élevage est quasi absent. La floriculture, quant à elle, se développe dans les différents sites, en marge des systèmes de production dominants et précisément dans les interstices urbains. Ce sont les migrants provenant de l'intérieur du pays en majorité qui en font leur métier. La production est destinée aux couches aisées et à la strate supérieure des couches moyennes des villes.

La production dans ces sites nécessite peu ou pas d'arrosage : l'alimentation en eau se faisant à partir de la remonté par capillarité des eaux de la nappe. La disposition des parcelles de cultures suit le tracé des berges et, s'étire en direction du centre de la vallée ou du lac. La taille des exportations dépasse rarement l'hectare. Sur la langue de barbarie, une technique assez originale permet aux maraîchers contraints par le manque de terres de remblayer les berges du fleuve ou, d'excaver les bordures dunaires afin d'étendre les surfaces cultivables. Les cultures sont essentiellement des cultures de décrue avec comme principales spéculations : le chou et la patate douce.

Les types d'exploitations

24 Les exploitations horticoles dans la région des Niayes sont de deux types: les exploitations maraîchères et les exploitations arboricoles.

Les exploitations maraîchères

Trois types d'exploitation maraîchère se distinguent dans la région des Niayes selon la taille et selon le mode de mise en valeur :

- Les petites exploitations : leur taille est inférieure à 1 hectare et relève plus d'exploitation individuelle que d'exploitation de type familiale. Ce caractère individuel reste très lié au mode d'appropriation et de mise en valeur de la terre. Il s'agit souvent de parcelles morcelées par leur propriétaire, lébou en général dans la région de Dakar, qui loue à des immigrés nationaux ou de la sous région particulièrement de la Guinée Conakry ou du confiage localement désigné sous le vocable de mbaye sëedo ou métayage. Ce type d'exploitation est dominant sur toute la bande des Niayes plus particulièrement dans les zones dépressionnaires et les vallées asséchées. Les spéculations qui s'y cultivent sont diverses et variées et sont essentiellement destinées à l'approvisionnement des marchés locaux. Contrairement aux autres régions, la production au niveau de Dakar est plus diversifiée et s'étale sur toute l'année.

- Les exploitations moyennes : leur taille est variable entre 1 et 20 hectares ; elles sont localisées sur les sols dior et, sur les vertisols dans la zone de Sébikotane et de Pout. Les exploitations sont privées, détenues par leurs propriétaires qui les ont acquises par héritage, par achat, par don ou, par location. De par leur mode de mise en valeur, ces exploitations sont de type moderne et semi-moderne faisant intervenir l'outil mécanique notamment dans le travail de la terre, l'exhaure de l'eau, des techniques d'irrigation et des ouvriers agricoles ou main 
d'œuvre salariée. Les spéculations sont moins diversifiées qu'au niveau des petites exploitations. La production étant essentiellement destinée au marché en gros et à l'exportation. C'est ainsi que les principales cultures sont l'oignon, la tomate, le haricot vert, le chou, l'aubergine, le jaxatu, le piment et le poivron. À elles seules, les petites et moyennes exploitations assurent $80 \%$ de la production maraîchère du pays.

- Les exploitations modernes : ce type d'exploitation dépasse 50 hectares et se caractérise par des moyens techniques et humains pour une rentabilité maximale. Elles sont privées ou à caractère associatif (GIE). Les exploitations modernes sont concentrées dans la région de Dakar (Sébikotane), Thiès (Pout, Mboro) et Saint-Louis. Leur production est constituée en majeure partie de culture d'exportation (haricots verts, tomates) ; les écarts de tri destinés à l'approvisionnement des marchés locaux.

Les exploitations arboricoles

De par leur taille, on distingue deux types d'exploitation :

- Les petites exploitations avec des tailles variables entre 0,5 et 2,5 hectares se trouvent à Saint-Louis précisément dans la zone de Sango spécialisée dans la production fruitière ; les espèces dominantes sont : cocotier, sapotier, manguier, papayer, corossolier, etc. Dans le reste de la zone dominent les agrumes et les mangues.

- Les grands exploitants dominent dans la région de Dakar et Thiès avec des surfaces supérieures à 5 hectares. Leur production est constituée davantage de mangues et d'agrumes. L'essentiel de la production de l'arboriculture dans la région des Niayes sert à l'approvisionnement des marchés locaux. Les petites exploitations inférieures à 1 hectare nombreuses dans la zone de Sango à St-Louis, sont gérées et exploitées par leurs propriétaires. Par contre, les grandes et moyennes exploitations sont gérées par des sourga salariés. Les propriétaires résident pour la plupart du temps en ville et ont souvent d'autres secteurs d'activité. On remarque souvent une association de l'arboriculture et du maraîchage dans ces exploitations.

Les spéculations

Les spéculations dans les Niayes sont des plus diversifiées. Cependant, cette diversification semble suivre une logique de spécialisation en fonction de la typologie des sols, de la région et, de la taille des exploitations. Ainsi, dans les zones dépressionnaires notamment les Niayes et les vallées asséchées caractérisées par leur exiguïté, des sols très riches et peu assujettis aux contraintes hydriques particulièrement dans la région de Dakar et de Thiès, les cultures dominantes sont le chou, l'oignon, le piment, l'aubergine, le jaxatu, la salade, la menthe, etc. Cette dernière joue un rôle important notamment en apport financier substantiel permettant le financement en intrants divers et, des besoins quotidiens des petits exploitants.

Au niveau des sols dior et les vertisols, sur lesquelles sont en général les moyennes et grandes exploitations, les cultures dominantes restent le haricot vert, l'oignon, la tomate, le gombo qui est plus cultivé durant la période de saison des pluies, le poivron etc. À ce niveau également on localise l'arboriculture particulièrement dans le triangle Sangalcam, Mbooro, Pout respectivement dans les régions de Dakar et Thiès avec une production essentiellement constituée d'agrumes, de mangues, de cocos et bananes. 
30 Elles reposent essentiellement sur les itinéraires techniques, sur l'exhaure et les méthodes d'irrigation. La maîtrise des itinéraires techniques dans la production horticole reste à parfaire même si d'une zone à une autre ou d'un type d'exploitation à un autre, il existe des différences. Ainsi, selon la spécialité de la région ou de la zone de production, les connaissances se limitent sur l'augmentation des rendements par l'accroissement des surfaces conditionné également par l'exhaure et les méthodes d'irrigation. La lutte phytosanitaire et les modes d'utilisation des produits agropharmaceutiques sont des plus précaires surtout au niveau des petites exploitations. Le problème est d'autant plus alarmant que les connaissances sur les rémanences des produits utilisés sont quasi-nulles et, les délais d'attente pour la dégradation de ces produits utilisés au hasard sont largement déterminés par les opportunités sur le marché.

31 Les techniques de fertilisation sont mal maitrisées parfois surtout en ce qui concerne l'utilisation des engrais minéraux et du compostage particulièrement chez les petits producteurs. En ce qui concerne la gestion de l'eau, trois types d'exploitation de l'eau existent dans les Niayes :

- L'exploitation par les "céanes" largement dominant surtout dans la frange proche littorale où la nappe phréatique est affleurante ou à une profondeur dépassant rarement trois mètres. Ce type d'exploitation de l'eau entraîne une irrigation manuelle à l'aide d'arrosoirs et de seaux. Cependant, dans les Niayes de Mboro, contrairement aux autres régions, une amélioration qui tend vers une mécanisation de ce système est perceptible. En effet, les "céanes" ont des envergures plus larges et les profondeurs sont augmentées. Ceci a permis d'avoir suffisamment d'eau et consécutivement une utilisation de la motopompe, une augmentation des surfaces cultivées et une utilisation de lance pour l'arrosage. Compte tenu de son originalité, nous avons désigné ce système sous le terme de "céanes améliorés".

- L'exploitation par les puits traditionnels est fréquente également dans les zones où la nappe est assez profonde. Elle nécessite également d'une manière générale une irrigation par arrosoir ou par seau. Ces deux types d'exploitation de l'eau, excepté le système des céanes améliorés, ne favorisent pas l'exploitation de grandes surfaces ; et le temps consacré à l'arrosage et au stockage de l'eau dans les bassins est long.

- l'exploitation par forage personnel ou adduction à partir du réseau de la SDE. Ces deux modes d'exploitation sont plus présentes dans la région de Dakar et Thiès notamment sur les axes Sangalcam, Mboro, Sébikhotane et Pout. La taille des exploitations est supérieure à 80 hectares dans les exploitations qui disposent de forages personnels. Toutefois, ces deux systèmes d'exploitation ont entraîné de nouvelles techniques d'irrigation notamment l'irrigation par aspersion et par goutte à goutte.

Interactions horticulture - élevage : potentiel du système et contraintes

L'horticulture est, à côté de la pêche, la principale activité productive pratiquée par les populations de la zone des Niayes au Sénégal. Une faible partie du cheptel national, de l'ordre de $3 \%$, est exploitée dans cette zone mais le mode de production est plus moderne que dans les zones extensives. Cette tentative de modernisation oriente le système de production vers une intensification très demandeuse d'intrants. Les producteurs tentent de répondre à cette demande par le recyclage de différents types de déchets. L'observation du système de production à travers les pratiques culturales et d'élevage et l'évaluation de ses performances suscite des interrogations relatives à la dynamique des processus d'interaction, les objectifs visés, le taux d'exploitation du potentiel et les contraintes rencontrées. 
Pratiques agricoles dans le système horticulture - élevage de la zone des NiayesAgriculture

Les techniques culturales pratiquées dans la zone des Niayes ne privilégient pas la mécanisation et le système n'est pas moderne. Le travail de la terre est essentiellement manuel dans la plupart des exploitations. Les autres opérations impliquent physiquement les acteurs à tous les niveaux depuis les semis jusqu'aux récoltes. La description du système agricole permet de constater la prévalence de l'utilisation du fumier comme engrais qui occupe une place importante dans le processus d'amendement des sols et constitue le premier maillon de l'intégration horticulture élevage. Le fumier est utilisé pour améliorer la texture des sols et leur pouvoir de rétention. Il leur apporte également la matière organique indispensable à la croissance des végétaux. C'est dire l'importance des déjections fécales récupérées dans les élevages de la zone des Niayes qui constituent un instrument majeur d'intégration entre l'horticulture et l'élevage.

L'utilisation de la matière organique est cependant contrainte par l'insuffisance du disponible et aussi par des problèmes d'accès (coût élevé + difficultés de transport). Sa qualité n'est pas optimale pour la plupart des cultures maraîchères qui requièrent son association à des engrais minéraux.

Elevage

35 L'élevage de la zone des Niayes a une dominante intensive ou se mi-intensive. Il héberge un faible pourcentage du cheptel national. Son extension s'oppose aux contraintes spatiales.

Les terres de parcours sont les plus sollicitées pour absorber les flux démographiques. Les Niayes hébergent également des troupeaux des zones environnantes. L'importation d'animaux des zones extensives vers les grandes agglomérations permet aux producteurs d'atteindre les grands marchés. Pour l'élevage des ruminants, il existe un système extensif avec pâturage dans les espaces résiduels, les couloirs des exploitations maraîchères. Ces animaux sont parqués la nuit. Le système semi-intensif fait intervenir des pratiques de supplémentation le soir après le pâturage. Le système intensif est très représenté. Il met en stabulation les animaux pour leur apporter les ressources alimentaires nécessaires pour la production de lait et/ou de viande. Ce système joue un important rôle dans l'approvisionnement des villes. C'est également une source d'emploi non négligeable pour des immigrants.

La zone des Niayes est la principale région avicole intensive du Sénégal. Son climat et la présence des marchés urbains ont favorisé l'implantation d'ateliers de production intensive de poulet et d'œuf. L'aviculture urbaine contribue beaucoup à l'intégration de l'horticulture et de l'élevage dans cette zone. La fiente de volaille est un engrais de choix dont l'appréciation du taux de satisfaction des besoins requiert une évaluation quantitative et qualitative.

38 L'utilisation des farines d'origine animale telles que la poudre d'os et la farine de sang pour l'alimentation du bétail est une autre possibilité exploitable dans le cadre d'une bonne interaction entre différentes spéculations agricoles. Les abattoirs produisent des déchets comme le sang, les os et le contenu digestifs qui pourraient être utilisés aussi bien dans l'enrichissement d'engrais organiques que dans l'alimentation du bétail. Cette dernière alternative est cependant limitée par les risques de contamination microbienne concernant la maladie de la vache folle notamment. La farine de poisson est déjà bien utilisée pour l'alimentation de la volaille. Les ateliers de transformation 
artisanale du poisson rejettent des déchets à base d'écailles et d'arêtes de poisson utilisé comme engrais.

Présence de l'arbre dans le système horticulture / élevage dans la zone des Niayes

La zone des Niayes recèle des espaces forestiers peu importants localisés à M'Bao et dans le Littoral atlantique. Ce sont des refuges et des niches alimentaires pour le bétail en divagation; elles représentent un premier niveau de recyclage des éléments nutritifs par dépôt de matière organique au sol et prélèvement des feuillages d'arbres par les ruminants. Les forêts fournissent également des feuilles d'arbres recyclables dans la préparation de fumier en association avec les déjections fécales des animaux domestiques pour l'amendement de surfaces maraîchères. Ces forêts souffrent de dégradations quelques fois très avancées, mais le plus grand danger est la déforestation au profit de l'extension des zones habitées. C'est tout le problème de la progression des villes au détriment de l'espace rural périurbain indispensable à un développement de l'agriculture urbaine.

Les phénomènes de dégradation et l'urbanisation n'ont pas épargné la strate arbustive de la zone des Niayes. Mais la présence d'espèces fourragères arbustives dans les exploitations horticoles permet de valoriser des terres exposées aux différentes formes d'érosion. Les arbres fourragers ont été récemment introduits dans certaines exploitations maraîchères dans la zone des Niayes. Le genre Leucaena en particulier est entrain de se propager dans la zone de Dakar. Leur introduction répond à des objectifs multiples : alimentation du bétail, amendement des sols, protection contre l'érosion éolienne et hydrique, délimitation de parcelles. Ces espèces ont contribué à améliorer les performances horticoles mais leur utilisation dans l'alimentation du bétail est encore embryonnaire. Leur installation est encore insuffisante, leur mise en place est à promouvoir.

\section{Productions halieutiques}

41 Entièrement bordée par le littoral atlantique, les Niayes couvrent la moitié des $718 \mathrm{~km}$ de côte maritime du Sénégal. Cette façade atlantique est poissonneuse et fait des Niayes la première région halieutique du Sénégal. La pêche représente la première activité économique des populations. Dans plusieurs zones des Niayes, les producteurs maraîchers associent d'ailleurs la pêche aux cultures maraîchères. Cette intégration offre des possibilités de recyclage. Les résidus de traitement des fruits de mer sont utilisés comme engrais sur tout le littoral atlantique. Ces résidus sont issus des ateliers de transformation artisanale de poisson.

Les principaux centres de débarquement sont Saint-Louis, Kayar, Hann. La transformation artisanale concerne le fumage, le salage et le séchage. 90\% des 130000 tonnes de poissons annuellement capturés au Sénégal sont traités et exportés. Leur traitement génère ainsi des sous-produits qui sont recyclés en direction de la production de farines d'origine animale mais aussi vers la production d'engrais. Des recherches s'avèrent nécessaires pour évaluer la qualité de ce produit et préciser son mode d'utilisation.

Flux des intrants et intégration horticulture - élevage

43 L'intensification de l'élevage et celle de l'agriculture s'impose comme la réponse la mieux adaptée à la contrainte spatiale qui limite l'expansion de l'agriculture urbaine au Sénégal. Cette alternative n'est pas sans conséquence sur la disponibilité des intrants qui s'avère comme le principal facteur qui limite les performances de l'horticulture et 
celle de l'élevage en zone péri urbaine. Ce système est déterminé par l'état des sols, le besoin d'amendement, et l'utilisation des résidus de culture.

Si les semences sont souvent d'un coût prohibitif, les engrais sont aussi d'accès difficile, ce qui oriente les producteurs vers l'utilisation des résidus d'élevage pour améliorer le statut des sols. Le cheptel reçoit un feed back en terme de résidus de cultures recyclables dans l'alimentation des ruminants et des animaux de trait. Quelles sont les caractéristiques et les contraintes de ce système qui illustre une solide interaction entre l'horticulture et l'élevage dans la zone des Niayes du Sénégal?

Etat et systèmes d'amélioration du statut des sols

Le Sénégal est peu doté en ressources pédologiques de qualité. Les principaux types de sols rencontrés sont de formations sableuses littorales constitués de cordons, de terrasses, de dunes du littoral et de la Grande Côte surtout, enserrant des sols argilo-sableux dans les bas fonds. Ils sont essentiellement constitués par des sols minéraux bruts peu fertiles parce que pauvres en limon et en matière organique.

Tableau 1. Principales contraintes à l'utilisation des terres dans la région des Niayes

\begin{tabular}{|l|l|l|}
\hline $\begin{array}{l}\text { Région } \\
\text { naturelle }\end{array}$ & agricole & Principales contraintes \\
\hline \hline Niayes & $\begin{array}{l}\text { - Insuffisances des pluies } \\
\text { - Menaces de l'avancée des dunes vives, remise en mouvement des dunes } \\
\text { anciennes } \\
\text { - Salinisation des sols et des puits } \\
\text { - Disparition de la végétation sur les dunes } \\
\text { - Comblement et ensablement des terres de bas-fond } \\
\text { - Risque de rupture d'équilibre entre eau douce et eau salée dans le sous-sol } \\
\text { et menace d'intrusion marine. }\end{array}$ \\
\hline
\end{tabular}

Le système d'amendement des sols : utilisation des engrais

De nombreux travaux sur la fertilisation des cultures maraîchères ont été réalisés dès 1961 à Bambey sur sol dior et se sont poursuivis dans la vallée et le Delta du fleuve Sénégal (sol alluvionnaire) et à N'diol (sol sableux dunaire), dans les Niayes, sur sol noir humifère plus ou moins salé et à Séfa (Sud de la Casamance) sur sol ferrugineux tropical lessivé à concrétions. Les principales recommandations démontrent comment sont exigeantes les cultures légumières. Ce qui pose un réel problème de disponibilité en intrants d'origine végétale ou animale.

Il faut signaler que la fertilisation minérale est abondamment utilisée dans la zone des Niayes comparée au reste du pays comme la zone du bassin arachidier. Les risques encourus pour la non-valorisation de l'engrais apporté lié au déficit hydrique des cultures céréalières résultant de la sécheresse ainsi que le faible pouvoir d'achat des paysans ont entravé l'utilisation des engrais minéraux. Un autre aspect est le coût prohibitif des intrants qui limite énormément son utilisation en milieu réel.

La quantité de fumier susceptible d'être collectée et valorisée pour les cultures à l'échelle de l'exploitation, du village, du terroir ou de la région dépend de l'effectif du bétail présent, de leur importance relative et de leur mode de gestion. Des enquêtes 
réalisés dans des villages (Diamsil-sessène) de la zone Nord du Bassin arachidier ont montré qu'un troupeau composé d'animaux de trait (30) et extensifs (407), produit annuellement respectivement 43 et 149 tonnes de matière sèche de fumier et $70 \% \mathrm{du}$ total sont produits en saison sèche (Sagna-Cabral, 1989). Des résultats comparables $(1,8$ tonnes de fumier par tête et par an) ont été obtenus par Fall et al., (1994) à Bambey alors que les animaux élevés en embouche bovine à Sébikotane ont donné des quantités de fumier plus importantes (3,5 tonnes par animal et par an). Ces résultats et les caractéristiques chimiques du fumier produit sont présentés dans le tableau 2 et indiquent la diversité des produits obtenus en milieu réel.

Importance de la fumure organique

Tableau 2. Caractéristiques chimiques du fumier produit dans 7 exploitations suivies à Ndiamsyl-sessène (Centre nord du bassin Arachidier)

\begin{tabular}{|l|l|l|l|l|l||l|l||l|}
\hline Exploitation & Nature du fumier & $\begin{array}{l}\mathrm{C} \\
\%\end{array}$ & $\begin{array}{l}\mathrm{N} \\
\%\end{array}$ & $\begin{array}{l}\mathrm{P} \\
\%\end{array}$ & $\begin{array}{l}\mathrm{K} \\
\%\end{array}$ & $\begin{array}{l}\mathrm{Ca} \\
\%\end{array}$ & $\begin{array}{l}\mathrm{Mg} \\
\%\end{array}$ & $\begin{array}{l}\text { Cendres insolubles } \\
\%\end{array}$ \\
\hline \hline 1 & Bovins & 34.3 & $\begin{array}{l}1.25 \\
2.25\end{array}$ & $\begin{array}{l}0.207 \\
0.224\end{array}$ & $\begin{array}{l}0.247 \\
0.570 \\
0.7\end{array}$ & $\begin{array}{l}1.53 \\
0.79 \\
2.02\end{array}$ & $\begin{array}{l}0.563 \\
0.597 \\
0.720\end{array}$ & $\begin{array}{l}22.1 \\
35.6 \\
25.7\end{array}$ \\
\hline \hline 2 & Ovin & 29.2 & 1.97 & 0.297 & 0.697 & \\
\hline \hline 3 & Cheval & 39.2 & 1.19 & 0.193 & 0.500 & 0.74 & 0.637 & 10.6 \\
\hline \hline 4 & Cheval & 31.5 & 0.87 & 0.153 & 0.160 & 0.69 & 0.403 & 25.4 \\
\hline \hline 5 & Chin & 34.3 & 2.04 & 0.254 & 0.500 & 2.31 & 0.613 & 24.2 \\
\hline \hline 6 & Cheval & 36.8 & 1.00 & 0.169 & 0.360 & 0.630 & 0.447 & 18.5 \\
\hline \hline 7
\end{tabular}

Le taux de cendres insolubles varie de 10 à $35 \%$; alors que celui des déjections fraîches est d'environ $5 \%$ (Ganry, et Badiane, 1998). Ce taux élevé de cendres insolubles diminue notablement la valeur fertilisante du fumier. L'action des termites et les mauvaises conditions de stockage qui induisent des pollutions diverses sont à l'origine de ce fait. La teneur en azote est correcte par contre les taux de phosphore et surtout de potassium sont faibles. Les taux de $\mathrm{Ca}$ et de $\mathrm{Mg}$, à l'exception du fumier ovin dont les valeurs sont relativement élevées ( $2 \%$ environ) ne présentent pas de différences notables par rapport aux valeurs couramment observées.

La grande dispersion des valeurs obtenues à l'analyse pour un même type d'animal, montre qu'il est nécessaire d'être prudent dans l'interprétation d'un échantillon aussi restreint, et souligne que la qualité minérale du fumier est très dépendante non seulement de l'alimentation de l'animal mais aussi des conditions de stockage dans l'exploitation. Par ailleurs, ces différences révèlent la possibilité voire la nécessité d'améliorer la qualité du fumier produit en milieu paysan pour accroître son efficacité. L'amendement des sols est souvent une nécessité pour des cultures maraîchères 
exigeantes en terme d'éléments nutritifs. Un certain nombre d'espèces, type manioc, pastèque, melon, semblent être plus sobres ; mais leur culture est un véritable facteur de détérioration des sols. La définition d'une stratégie de régénération des sols est une question clé à adresser pour améliorer les rendements agricoles dans la zone des Niayes.

Utilisation des résidus de cultures maraichères pour l'alimentation du bétail

51 La récolte des cultures maraîchères laisse sur le champ une biomasse utilisée par le producteur pour nourrir le bétail. L'importance des résidus de cultures maraîchères pour l'alimentation du bétail est reconnue par les producteurs. Le disponible en résidus de récoltes provenant des cultures maraîchères n'est pas maîtrisé. Il peut s'estimer à partir de la mesure du rapport produit / sous-produit. Ce rapport montre la supériorité des résidus comparés aux produits. Une évaluation préliminaire des résidus horticoles est présentée au tableau 4.

Pour certains produits l'état actuel des statistiques agricoles ne permet pas d'estimer la production nationale. La fane de haricot semble être le produit de plus grande valeur. Elle est totalement récupérée et fait l'objet d'un marché à l'échelle nationale. Les quantités produites dans la vallée du Fleuve Sénégal sont par exemple acheminées vers la région de Dakar pour l'alimentation du cheptel urbain. La paille d'oignons est également très prisée, elle est ramassée par les producteurs pour l'alimentation des moutons de case.

53 Les autres sous-produits horticoles ont un faible taux d'utilisation. On observe une trop grande déperdition de résidus qui sont brûlés, enfouis ou dispersés par les vents sur les champs. Les producteurs n'élèvent pas souvent des animaux pour assurer le recyclage de cette biomasse. Le problème du transport et de la conservation de ces sous-produits est une des contraintes à leur utilisation.

54 Les données sur la composition chimique et la valeur nutritive des sous-produits sont insuffisantes. Mais l'expérience des éleveurs reconnait la haute valeur nutritive des fanes de haricot et d'oignons, des résidus de culture de manioc et de la tomate.

55 Le tableau 3 donne la valeur nutritive de quelques résidus. La fane de pomme de terre est comparable à un foin de bonne qualité alors que les résidus de patate, de haricot et de manioc sont plus proches de concentrés type son de mil et graine de coton.

Production de fourrage au tour des céanes pour l'alimentation du cheptel urbain

56 La prolifération d'espèces herbacées de nature graminéenne autour des céanes permet au producteur d'exploiter cette biomasse comme fourrage. L'herbe est commercialisée sur le marché des villes pour alimenter le cheptel urbain. Cette activité peut apporter 2500 à 5000 FCFA par jour au collecteur itinérant. Les quantités de ce produit et l'importance de cette filière sont à évaluer.

Traction animale

57 La traction animale est un phénomène très répandu dans la zone périurbaine des Niayes. Elle s'explique par l'existence d'un réseau routier assez dense mais insuffisamment ramifié pour atteindre des zones de production reculées. Les particularités topographiques caractérisées par l'existence de dunes de sables limitent également l'accès des périmètres maraîchers aux véhicules. L'animal n'intervient pas dans le travail de la terre mais il est souvent la seule alternative susceptible d'apporter une réponse à l'épineux problème du transport des intrants, du déstockage des produits et de la mobilité du producteur. 
Tableau 3. Qualité des résidus de cultures maraîchères

\begin{tabular}{|c|c|c|c|c|c|c|}
\hline Espèces & MS & $\mathrm{CB}$ & MAT & UF & $\mathrm{Ca}$ & $\mathrm{P}$ \\
\hline \multicolumn{7}{|l|}{$n-g / k g M S$} \\
\hline \multicolumn{7}{|l|}{ Oignon } \\
\hline Tomate & & & & & & \\
\hline Pomme de terre & 194 & 248 & 134 & 0.55 & 25.7 & 2.3 \\
\hline Patate* & 140 & 174 & 157 & 0.88 & 9.1 & 5.4 \\
\hline Patate & 158 & 177 & 108 & 0.71 & 18.8 & 1.0 \\
\hline Haricot (séchés) & 964 & 214 & 231 & 0.77 & 19.6 & 3.1 \\
\hline \multicolumn{7}{|l|}{ Carotte } \\
\hline \multicolumn{7}{|l|}{ Choux } \\
\hline Manioc* & 210 & 157 & 273 & 1.05 & 13 & 5.6 \\
\hline Manioc & 273 & 215 & 156 & 0.64 & 9.2 & 5.1 \\
\hline
\end{tabular}

Source : Kearl (1982) - Rivière (1978)

58 Les espèces animales utilisées en priorité sont les équins et les asins; la taille de ce cheptel est faible allant de 1 à 5 unités par exploitation (Fall et al., 1994). La gestion du cheptel de traction animale ne fait pas l'objet d'une planification particulière. Le parcage se fait dans les arrières cours des habitations et l'alimentation avec les résidus de cultures maraîchères. Il n'existe pas de prophylaxie contre les principales maladies sévissant dans la zone.

Le développement de la traction animale est limité par des contraintes liées au système d'exploitation du cheptel de traction. La charge de travail est souvent excessive. Les carences alimentaires ont une forte pré valence. On ne note pas à proprement parler l'existence d'un plan d'alimentation. L'animal bénéficie de restes éventuels. Les monogastriques en particulier ont peu accès aux céréales qui devraient constituer une importante partie de leur régime alimentaire. Le suivi sanitaire fait défaut sauf en cas de flambées épizootiques.

60 Les animaux de trait ont en charge tout le travail de transport dans les zones horticoles. Il s'agit du transport des producteurs et des produits, celui des intrants et la traction du matériel de labour. Leur rôle est donc incommensurable dans l'accès aux intrants, le travail de la terre et le déstockage des produits notamment leur conduite du bord champ au marché primaire. Ces animaux sont plus adaptés à l'enclavement et à la mauvaise qualité des routes. Leur intervention n'arrive cependant pas encore à régler de façon satisfaisante le problème de la distribution des intrants et des produits. Dans certaines zones, notamment dans la région de Louga et dans le Gandiolais, les problèmes de transport de la production et l'absence de structures de conservation sont les principales causes de pertes par détérioration des produits.

Potentiel inexploité dans le domaine de l'intégration horticulture - élevage dans la zone des NiayesOptimisation de l'utilisation de la matière organique pour l'amendement des sols 
61 La zone des Niayes héberge 3\% du cheptel national soit 33100 bovins, 179000 ovins et caprins, 17100 équins et asins et 5220000 unités de volailles. Quoique numériquement faible comparé aux autres régions, ce cheptel pourrait davantage contribuer et de façon significative à l'amendement des sols. Sa gestion en stabulation pourrait ainsi favoriser le récupération et la gestion des déjections fécales. Les données préliminaires disponibles au LNERV (Fall et al., 1994) permettent en ce qui concerne les bovins d'évaluer à 115850 tonnes par an la quantité de fumier produite. Ces quantités pourraient amender près de 11585 ha (à raison de 10 tonnes par hectare) ou autant d'exploitations si on considère que la majorité des petits exploitants ont une surface moyenne de l'hectare. En d'autres termes, il serait possible, grâce à une gestion rationnelle $\mathrm{du}$ fumier de bovins de procéder à la satisfaction des besoins en matière organique de la totalité des surfaces fruitières et maraîchères de la zone des Niayes.

Des travaux de recherche devraient tenter d'évaluer les quantités de fumier produites chez les petits ruminants, les équidés et la volaille. Mais force est de constater que ce potentiel n'est pas utilisé à bon escient. L'accès au fumier est encore limité par une gestion extensive à semi-intensive du cheptel, associé à un taux de déperdition quantitative et qualitative non négligeable du fumier.

Le flux de résidus de culture comme ressource alimentaire pour le bétail

La quantification des résidus de récoltes d'origine maraîchère dans la zone des Niayes est en cours mais on peut dire que leur biomasse est importante. Cette matière végétale n'est pas utilisée à bon escient. Pourtant elle aurait pu aider davantage à lever les contraintes alimentaires qui limitent les performances des ruminants dans une zone de pâturage réduit et, résoudre le problème de l'alimentation d'un cheptel de trait indispensable.

Elle aurait pu également promouvoir une diversification pastorale dans le sens du développement de la cuniculture et de l'élevage d'animaux de bassecour comme les dindes et canards sans oublier les possibilités de vente de fourrage dans le marché urbain pour alimenter un cheptel urbain assez important.

Utilisation des ressources alimentaires non-conventionnelles pour le bétail

Les sous-produits d'abattoirs ne font pas l'objet d'une collecte et d'une transformation adéquates pour être recyclé dans l'alimentation du bétail. Il s'agit de récupérer et de traiter les os, le sang et le contenu digestif issu des abattoirs. Pourtant ils représentent un potentiel très bien utilisé dans les pays développés jusqu'à un passé récent. Aujourd'hui, les risques de contamination par la maladie de la vache folle limitent les possibilités de leur exploitation. Mais il serait possible de recycler les éléments nutritifs qu'ils apportent dans l'horticulture.

Contraintes a l'intégration horticulture élevage

66 Les possibilités d'intégration entre l'horticulture et l'élevage dans la zone des Niayes sont limitées par de nombreuses contraintes.

67 Le système de gestion de la terre ne facilite pas l'accès au petit exploitant alors qu'il ne favorise pas l'investissement. Les terres de haute valeur font l'objet d'une forte spéculation dans la zone des Niayes que la loi sur le domaine national n'arrive pas à contrôler. Cette situation est aggravée par une forte compétition qui souvent favorise l'urbanisme aux détriments de l'agriculture urbaine. L'expansion démographique renforcée par l'exode rural aggrave ainsi les difficultés d'accès à la terre. La majorité 
des exploitations sont de petite taille; leur surface est insuffisante pour intégrer l'agriculture et l'élevage in situ.

Le développement de l'élevage se heurte à un grand problème de sécurité à l'échelle du terroir et l'exploitation dans la zone des Niayes. En effet, l'élevage d'animaux domestiques dans la zone des Niayes comporte des risques de vol. Les exploitations sont peu protégées par des haies vives insuffisamment étanches. Le gardiennage est coûteux mais indispensable à la protection des investissements agricoles et pastoraux. D'ailleurs la plupart des exploitations ne sont pas mixtes. La contraintes liée à la sécurité apparaît comme une des premières causes de non-intégration entre l'horticulture et l'élevage dans la zone des Niayes.

Contrairement aux agriculteurs du bassin arachidier, les horticulteurs de la zone des Niayes intègrent moins l'élevage à leurs activités. On note une spécialisation plus poussée en direction de l'agriculture même si les deux activités sont en présence sur le terrain. L'existence du métayage ou la promotion de l'emploi agricole ne favorisent pas la diversification des activités allant dans le sens de l'implantation de l'élevage à coté des périmètres horticoles. Les acteurs non-propriétaires d'exploitations ne font pas des investissements durables. Les "agriculteurs du dimanche" ne s'engagent pas non plus fermement dans la voie vers l'intégration.

70 La commercialisation est une contrainte qui limite le développement des filières horticoles et pastorales. L'écoulement des produits périssables n'est pas complet. Le prix au producteur insuffisamment rémunérateur. Le caractère cyclique de la production entraîne un défaut de couverture de la période de contre-saison.

71 L'association horticulture/élevage n'étant pas une pratique répandue à l'échelle exploitation dans la zone des Niayes, il se pose un problème de transport des sous-produits vers les élevages. Cette opération est réalisée par les charrettes avec un certain taux de déperdition. Comme les fruits et légumes frais, les sous-produits de culture maraîchère ont une forte humidité. Il est souvent nécessaire de procéder à une rapide conservation avant détérioration. Le séchage est la méthode de conservation la plus répandue. Les sous-produits ne sont pas cependant protégés ce qui est la cause d'importantes pertes de biomasses.

Les technologies intégrant l'horticulture à l'élevage n'ont pas fait l'objet d'une vulgarisation satisfaisante. En ce qui concerne l'amendement des sols il se pose un problème de maitrise de la qualité et des modes d'utilisation du produit, les techniques d'association avec l'engrais minéral et la préparation du compost. Sur le terrain, il existe une demande d'encadrement. L'utilisation de résidus de récolte nécessite des recommandations sur les résidus utilisables, leur évaluation quantitative, les méthodes d'utilisation et les risques de toxicité. La traction animale demande également des investigations pour une amélioration de la gestion du troupeau de trait et du matériel de traction.

73 Certaines technologies n'ont pas fait l'objet de diffusion auprès des producteurs. D'autres ont connu de faibles taux de passage. Les causes du faible taux de passage des technologies sont multiples. Si leur inadéquation ou inadaptation peuvent être les principales causes de faible performance, la faiblesse du dispositif d'information des utilisateurs est un facteur qui limite leur diffusion.

Conclusion 
74 La question posée concerne la viabilité du système agricole périurbain et son impact sur l'expansion des villes. Quel rôle peuvent jouer les interactions horticulture-élevage pour limiter l'étendue du risque écologique? L'agriculture urbaine est une donnée permanente dans des villes en expansion. Nous avons observé une solide implantation des différents domaines de l'agriculture en ville dans l'espace périurbain. Dans la zone de Dakar, le système pastoral intensif s'implante et se consolide depuis plus d'une dizaine d'années. Le cheptel intensif présent est minoritaire numériquement, mais les rendements de production de viande et de lait y sont plus importants que partout ailleurs au Sénégal. En ce qui concerne l'horticulture, les espaces interstitiels périurbains sont de fait une importante partie des surfaces cultivées qui produisent un gros tonnage de légumes destinés à l'approvisionnement des villes. Les acteurs sont composés de migrants venant des zones rurales ou même de pays limitrophes mais en contrats avec des résidents qui tirent leurs moyens de subsistance du métayage. En ville, l'élevage de moutons et d'animaux de la bassecour est une des caractéristiques sociales qui marque les familles sénégalaises. Ce cheptel urbain est à prendre en compte dans l'évaluation des apports en terme de ressources alimentaires ou de revenus mais aussi à travers le rôle social que joue l'élevage.

On peut donc affirmer que l'agriculture urbaine est une activité qui représente une importante source de revenus pour les populations urbaines et suburbaines. De fait, l'agriculture urbaine s'est bien implantée; il convient alors de l'accepter et de ne pas marginaliser les populations qui en tirent leur revenu ou minimiser sa contribution à l'approvisionnement des villes. Mais le développement de ce secteur nécessite une bonne évaluation et un contrôle du risque écologique puis un bon arbitrage entre activités pour une utilisation harmonieuse de l'espace.

La zone de Saint-Louis est la moins consommatrice de produits chimiques. La zone de Dakar et celle de Thiès présente des risques écologiques évidents. Les produits chimiques ne sont pas utilisés à bon escient. Il semble difficile pour des acteurs en majorité illettrés de cerner le danger que présentent ces produits chimiques. Une gamme importante de produits pesticides est utilisée dans l'agriculture maraîchère dans la zone des Niayes.

Dans cette étude exploratoire, près de 123 produits ont été identifiés dont la moitié dans la région de Dakar. Cette région est la plus forte consommatrice de produits chimiques. Thiès et Saint-Louis en consomment modérément alors qu'à Louga, une telle consommation est quasi absente. L'utilisation des eaux usées est un danger préoccupant dans l'espace périurbain de la zone de Dakar. Les principaux canaux d'évacuation des eaux usées subissent des dérivations vers les champs. La grande Niayes de Pikine el les périmètres de Cambérène semblent être les zones les plus affectées par cette pratique.

Une insuffisance d'intégration entre l'horticulture et l'élevage a été observée. Ces relations sont plus dynamiques dans le sens des apports de l'élevage vers l'horticulture. Le feed-back est à stimuler pour que ces interactions soient la voie prioritaire pour contourner les difficultés d'accès aux intrants chimiques et assurer un bon recyclage des déchets dans les villes. Ces interactions horticulture - élevage représentent donc une solution à promouvoir pour assurer une utilisation judicieuse des nutriments à l'échelle terroir et exploitation. 


\section{BIBLIOGRAPHIE}

Fall S.T., Traoré E., Cissé M., Koréa A., Sèye B.M, Fall A., Diop A.K. 1994. Systèmes d'alimentation pour la production intensive de viande au Sénégal ; Essais de pré vulgarisation de rations d'embouche. Rapport Technique ISRA LNERV Réf W 30. Res Alim Décembre, 24 p.

Ganry F, Badiane A. 1998. "La valorisation agricole des fumiers et compost en Afrique soudano-sahélienne". Diagnostic et Perspectives. Agriculture et Développement : Spécial sol tropicaux, $n^{\circ} 18: 73-80$.

Kearl L.C. 1982. Nutrient requirements of ruminants in the developping countries. Internationnal Feedstuff lnstitute Publ. Utah Agriculture Experiment station. Utah State University, Logan, 381p.

Mougeot J.A. et Sawio Camillus J. 1995. "Faire Campagne en ville. L'agriculture urbaine en Afrique de l'est". CRDI : 57 - 78.

Mougeot J.A. et Sawio Camillus J. 1995. "Faire Campagne en ville. L'agriculture urbaine en Afrique de l'est". CRDI : 1 - 30.

Mbaye A., Moustier P. 2000. "Market-oriented urban agricultural production in Dakar", in : Growing Cities, Growing food. Urban agriculture on the policy agenda, Bakker N., Dubbeling.

Riviere R. 1978. "Manuel d'alimentation des ruminants domestiques en milieu tropical". Manuels et Précis d'Elevage (W9). Paris, Min. Coop. : 472-481.

Sagna-Cabral M.A. 1989. Utilisation et Gestion de la matière organique d'origine animale dans un terroir du centre-nord Sénégal. Cas du village de Ndiamsil-Seesène. Mémoire d'Etudes. ENSAAA de DIJON / CNEARC- ENSAT, 45p. + annexes.

\section{NOTES}

1.Mboro est un important marché-relais d'où partent les produits maraîchers vers, par ordre d'importance numérique. Dakar. Mauritanie. Touba. Ngaye-Mekhé. Touba Toul. Thiès. Kaolack. Il concentre $70 \%$ de la production légumière de toute la région de Thiès qui. à son tour. réalise $40 \%$ de la production nationale.

\section{AUTEURS}

\section{SAFIÉTOU TOURÉ FALL}

Dr. Vétérinaire - Zootechnicienne, spécialiste en Nutrition - ISRA

\section{ABDOU SALAM FALL}

Sociologue et Anthropologue - IFAN-Université Cheikh Anta Diop de Dakar 
IBRAHIMA CISSÉ

Géographe-Environnementaliste - Université Cheikh Anta Diop de Dakar (UCAD)-Département de Géographie.

\section{AMINATA BADIANE}

Agronome-Pédologue - Direction Scientifique Institut Sénégalais de Recherches

Agricoles (ISRA)

\section{CHEIKH ALASSANE FALL}

Agronome, ISRA, Centre de Développement de l'Horticulture (CDH)

\section{MATY BA DIAO}

Agronome-Zootechnicienne - ISRA - Centre de Développement de l'Horticulture (CDH) 